\title{
DESIGN OF SPACE-TIME TRELLIS CODES FOR FULL SPATIAL DIVERSITY
}

\author{
Zoltan Safar and K.J. Ray Liu \\ Department of Electrical and Computer Engineering \\ University of Maryland at College Park \\ College Park, MD 20742
}

\begin{abstract}
Space-time codes have been developed to answer the need for high data rates in future wireless communication systems. In this work, we propose a systematic code construction method that provides full diversity advantage for any number of transmit antennas, any number of encoder states, and any constellation. Our approach is to exploit the properties of the state transitions in the trellis to force the desired structure on the channel symbol difference matrix. The design rules do not specify the space-time codes completely, leaving room for further optimization for coding advantage.
\end{abstract}

\section{INTRODUCTION}

In wireless communications, diversity techniques are widely used to combat the adverse effects of the radio propagation environment. For example, channel coding, a form of time diversity, adds redundancy with a certain algebraic structure that can be exploited to detect and correct transmission errors. Spatial diversity corresponds to redundancy in the spatial domain: building a system with multiple transmit and/or receive antennas can improve the quality of the wireless link by making use of the larger number of propagation paths between the transmitter and the receiver.

Space-time (ST) trellis codes represent a combination of forward error correction, transmit diversity and modulation. References [1] and [2] derived the performance criteria that characterize the ST codes with two quantities: the diversity advantage, which describes the asymptotic error rate decrease as a function of the signal to noise ratio (SNR), and the coding advantage, which determines the vertical shift of the error performance curve. The authors of [2] proposed design rules for two transmit antennas to achieve the maximum diversity advantage. They also derived a lower bound on the complexity of the encoder and the decoder for the desired diversity advantage and data throughput. This lower bound states that in order to achieve a diversity advantage of $K$ and to transmit one $B$-ary source symbol per state transition, the encoder and the decoder must have at least $N_{\min }=B^{K-1}$ states.

The repetition coded delay diversity scheme of [3] was the first systematic design rule for arbitrary number of transmit antennas. Using this method, ST codes achieving full diversity advantage can be designed for arbitrary constellations and encoders with $N_{\text {min }}$ states. They also introduced the idea of zero symmetry to constrain computer search for ST codes with more than two antennas.

The authors of [4] transformed the design problem into binary domain. They proposed design methods for an arbitrary number of transmit antennas and an arbitrary number of states, but only for BPSK and QPSK constellations. Moreover, the design methods of
[3] and [4] for full diversity advantage uniquely determine the ST codes, not leaving room to improve the coding advantage.

In this paper, we propose a systematic design method based on an alternative approach: we exploit the structure of the trellis to design ST codes that provide full diversity advantage for arbitrary number of transmit antennas, arbitrary power of two number of encoder states (as long as it satisfies the lower bound) and arbitrary constellations. Our method can be treated as a generalization of the results of [2] and [3]. The design rules for full diversity advantage do not specify the ST codes completely, offering the possibility to further optimize for coding advantage.

The paper is structured in the following way. In Section 2, we will describe the system model and introduce the notation used in this paper. The design criteria derived in [1] and [2] will be briefly restated in Section 3. The development of our design method will also be described in this section. Section 4 will provide the simulation results, and some conclusions are drawn in the last section.

\section{SYSTEM MODEL}

Consider a wireless communications system with $K$ transmit and $L$ receive antennas. The input bit stream is divided into $b$ bit long blocks, forming $B$-ary $\left(B=2^{b}\right)$ source symbols. The ST encoder works as a finite state machine with $N$ states: it takes the current source symbol, $b_{t}\left(b_{t} \in\{0,1, \ldots, B-1\}\right)$ at discrete time $t$, and governed by this input and the current state, $S_{t}$ $\left(S_{t} \in\{0,1, \ldots, N-1\}\right)$, it moves to the next state, $S_{t+1}$. During this state transition, the encoder outputs $K B$-ary channel symbol indices. We denote by $i^{k}\left(S_{t}, b_{t}\right)$ the channel symbol index for antenna $k, k=0,1, \ldots, K-1$, generated during the state transition from $S_{t}$ when the current source symbol is $b_{t}$. We will also use the channel symbol index vector, defined as:

$$
\mathbf{i}\left(S_{t}, b_{t}\right)=\left[i^{0}\left(S_{t}, b_{t}\right), i^{1}\left(S_{t}, b_{t}\right), \ldots, i^{K-1}\left(S_{t}, b_{t}\right)\right]^{T} .
$$

These channel symbol indices are mapped onto channel symbols (or constellation points) by the modulators and transmitted through the transmit antennas. In the sequel, $c(i)$ will represent the constellation point corresponding to channel symbol index $i$. (For example, in case of $B$-ary PSK, $c(i)=\exp (j 2 \pi i / B)$, where $j=\sqrt{-1}$.) All the constellations are assumed to be normalized so that the average energy of the constellation is one (if the channel symbols are equally likely). $c\left(i^{k}\left(S_{t}, b_{t}\right)\right)$ will denote the constellation point output by antenna $k$ when the current state is $S_{t}$ and the current input is $b_{t}$. In vector notation:

$\mathbf{c}\left(S_{t}, b_{t}\right)=\left[c\left(i^{0}\left(S_{t}, b_{t}\right)\right), c\left(i^{1}\left(S_{t}, b_{t}\right)\right), \ldots, c\left(i^{K-1}\left(S_{t}, b_{t}\right)\right)\right]^{T}$. 
The transmission medium is assumed to be flat (frequency nonselective), quasi-static Rayleigh fading channel. The quasistatic property means that the channel remains constant over a certain time, called the frame period, and changes independently from one frame to the other.

Furthermore, some additional assumptions are made. First, the receiver has knowledge of the path gains between the transmit and the receive antennas. They are modeled as independent, complex, zero mean, circularly symmetric Gaussian random variables with unit variance. Second, the receiver is perfectly synchronized with the transmitter.

At the receiver side, the received signals at each receive antenna are demodulated, and the ST decoder produces the decoded bit stream. The receiver noise is modeled as independent, complex, zero mean, circularly symmetric Gaussian random variables.

\section{DESIGN FOR DIVERSITY ADVANTAGE}

Assume that the previously decribed transmitter sends $T(T>$ $K) B$-ary source symbols to the receiver. The ST encoder, while encoding the data, goes through the following sequence of states:

$$
S_{0} \stackrel{b_{0}}{\longrightarrow} S_{1} \stackrel{b_{1}}{\longrightarrow} S_{2} \stackrel{b_{2}}{\longrightarrow} \ldots \stackrel{b_{T-2}}{\longrightarrow} S_{T-1} \stackrel{b_{T-1}}{\longrightarrow} S_{T} .
$$

In words, the encoder starts in $S_{0}$, takes the first input $b$-tuple, $b_{0}$, moves to $S_{1}$, and so on. As a result of this state transition sequence, the encoder produces the channel symbol vector sequence:

$$
\mathbf{c}\left(S_{0}, b_{0}\right), \mathbf{c}\left(S_{1}, b_{1}\right), \ldots, \mathbf{c}\left(S_{T-1}, b_{T-1}\right) .
$$

The above vector sequence can be arranged into a $K$ by $T$ matrix, C:

$$
\mathbf{C}=\left[\mathbf{c}\left(S_{0}, b_{0}\right), \mathbf{c}\left(S_{1}, b_{1}\right), \ldots, \mathbf{c}\left(S_{T-1}, b_{T-1}\right)\right] .
$$

The decoder, due to decoding errors, goes through a different sequence of states,

$$
S_{0}^{\prime} \stackrel{b_{0}^{\prime}}{\longrightarrow} S_{1}^{\prime} \stackrel{b_{1}^{\prime}}{\longrightarrow} S_{2}^{\prime} \stackrel{b_{2}^{\prime}}{\longrightarrow} \ldots \stackrel{b_{T-2}^{\prime}}{\longrightarrow} S_{T-1}^{\prime} \stackrel{b_{T-1}^{\prime}}{\longrightarrow} S_{T}^{\prime},
$$

producing the erroneously decoded source symbol sequence $\left\{b_{t}\right\}$ and the $K$ by $T$ channel symbol matrix $\mathbf{C}^{\prime}$ :

$$
\mathbf{C}^{\prime}=\left[\mathbf{c}\left(S_{0}^{\prime}, b_{0}^{\prime}\right), \mathbf{c}\left(S_{1}^{\prime}, b_{1}^{\prime}\right), \ldots, \mathbf{c}\left(S_{T-1}^{\prime}, b_{T-1}^{\prime}\right)\right] .
$$

We can define $\mathbf{B}$, the channel symbol difference matrix as: $\mathbf{B}=\mathbf{C}-\mathbf{C}^{\prime}$ and a $K$ by $K$ matrix $\mathbf{A}$ as: $\mathbf{A}=\mathbf{B B}^{H}$. The design criteria [1],[2] for the earlier described channel model were derived to minimize the probability that the decoder erroneously decodes $\mathbf{C}^{\prime}$ if $\mathbf{C}$ was sent

1. Design for full spatial diversity (rank criterion): The matrix A (or equivalently, the matrix B) must be of full rank for any distinct $\mathbf{C}$ and $\mathbf{C}^{\prime}$ matrices.

2. Design for coding advantage (determinant criterion): The minimum determinant of $\mathbf{A}$ taken over all distinct $\mathbf{C}$ and $\mathbf{C}$ matrices must be as large as possible. If the minimum determinant is $\gamma$, then a coding advantage of $\sqrt[k]{\gamma}$ has been achieved.

The design criteria described above do not provide a systematic method to construct ST trellis codes. In what follows, we propose design rules that guarantee that the resulting ST code achieves full spatial diversity.

In the ST encoder, $B$ channel symbol index vectors are assigned to each state, according to the branches emanating from

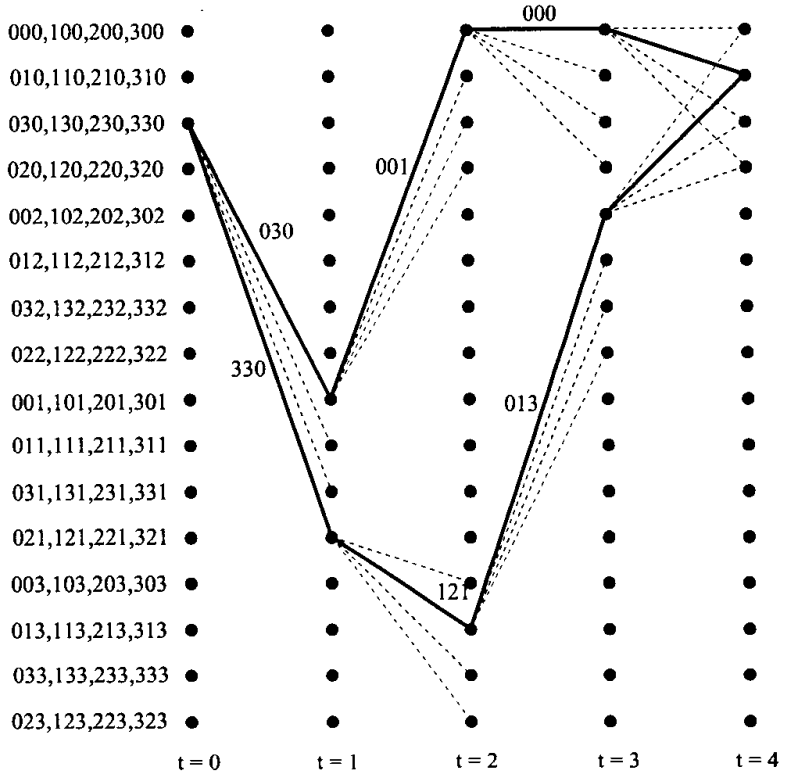

Fig. 1. Example ST code for 3 antennas, QPSK

that state. The current source symbol selects one of them, and the $k$ th $(k=0,1, \ldots, K-1)$ index of the chosen vector determines the constellation point for antenna $k$. Figure 1 depicts an example ST code for 3 antennas and QPSK constellation $(K=3, N=$ $16, b=2$ ). In this case, if the current state is state 2 and the value of the current source symbol is 3 (binary 11), the ST encoder selects the 3 rd channel symbol index vector, $[3,3,0]^{T}$, and moves to state 11 . The 0 th, 1 st and 2 nd antennas will transmit the channel symbols corresponding to the indices 3,3 and 0 , respectively.

Assume that the encoder has $N=R B^{K+p-1}$ states with $R=$ $2^{r}, B=2^{b}, b>0, p \geq 0$ and $0 \leq r<b$. Any power of two $N \geq N_{\min }$ can be put in this form. The state transition of the encoder at time $t$ is given by:

$$
S_{t}=\left(B S_{t-1}+b_{t-1}\right) \bmod N .
$$

By unfolding the recursion, for $1 \leq t \leq K+p-1, S_{t}$ can be expressed as:

$$
S_{t}=B^{t}\left(S_{0} \bmod \left(R B^{K+p-t-1}\right)\right)+\sum_{m=0}^{t-1} B^{t-1-m} b_{m} .
$$

Without loss of generality, we can assume that the first decoding error occurs at state $S_{0}$, so the correct and erroneous paths diverge at $S_{0}$. As a consequence, we have $S_{0}=S_{0}^{\prime}$ and $b_{0} \neq b_{0}^{\prime}$. From Equation (1), we can conclude that for arbitrary $p \geq 0$ and $0 \leq r<b$, the shortest length error path is at least $K$ long (i.e. for $t=1,2, \ldots, K-1, S_{t} \neq S_{t}^{\prime}$ ). The paths diverging at $S_{0}$ can merge only at $S_{K}$ or later.

For now, we are concerned only about the first $K$ long segment of all error paths of length $K$ or larger immediately after the first error event has occured. Our goal is to construct the $K$ by $K$ channel symbol difference matrix $\mathbf{B}_{\mathbf{1}}$, defined as

$$
\begin{aligned}
\mathbf{B}_{1}=\left[\mathbf{c}\left(S_{0}, b_{0}\right)-\mathbf{c}\left(S_{0}^{\prime}, b_{0}^{\prime}\right), \ldots\right. \\
\left.\ldots, \mathbf{c}\left(S_{K-1}, b_{K-1}\right)-\mathbf{c}\left(S_{K-1}^{\prime}, b_{K-1}^{\prime}\right)\right],
\end{aligned}
$$


in such a way that it is of full rank for any possible correct and errorneous paths through the trellis. Our method is to make $\mathbf{B}_{1}$ upper triangular with nonzero diagonal elements. As we go state transition by state transition in the trellis from $S_{0}$ to $S_{K}$, the design rules will force the desired structure on the $B_{1}$ matrix.

The $S_{0} \rightarrow S_{1}$ state transition is special since both the correct and the erroneous paths start at the same state. The goal is to set the 0 th entry of the 0th column of $\mathbf{B}_{\mathbf{1}}$ to a nonzero value and to zero out the rest of the entries in that column. This can be achieved by the following conditions that form the first half of the design rules:

(1a) The 0 th indices of the channel symbol index vectors at the same state must be different.

(1b) The remaining indices of the channel symbol index vectors at the same state must be the same.

In our example, assume that the $b_{0}=0$ (top) path is the correct path and the $b_{0}^{\prime}=3$ (bottom) path is the erroneously decoded path. The channel symbol index vectors $[0,3,0]^{T}$ and $[3,3,0]^{T}$ have different 0 th indices, but the 1st and 2 nd indices are the same; therefore, the 0th column of the $\mathbf{B}_{1}$ matrix will be $[1+j, 0,0]^{T}$.

For the rest of the state transitions $S_{t} \rightarrow S_{t+1}, t=1,2, \ldots, K-$ 1 , the objective is to set the $t$ th entry of the $t$ th column of $\mathbf{B}_{\mathbf{1}}$ to a nonzero value and to zero out all the entries below the $t$ th entry in that column. The method will be explained with the aid of the definitions given below:

Definition 1: A level t group is a collection of all possible destination states that can be reached from a given $S_{0}$ starting state at state transition $t$ for any $b_{0}, b_{1}, \ldots, b_{t-1}$ input sequence.

Definition 2 : A subgroup of a level $t$ group is a collection of all possible destination states that can be reached from a given $S_{0}$ starting state through a given $b_{0}$ starting branch in the trellis at state transition $t$ for any $b_{1}, b_{2}, \ldots, b_{t-1}$ input sequence.

Based on Equation (1) and the above definitions, we can establish the following facts for $t=1,2, \ldots, K-1$ :

1. Any level $t$ group starts at state $m$ such that $m \bmod B^{t}=0$ and consists of $B^{t}$ consecutive states.

2. Any subgroup of a level $t$ group starts at state $m$ such that $m \bmod B^{t-1}=0$ and consists of $B^{t-1}$ consecutive states.

3. Every level $t$ group consists of $B$ disjoint subgroups.

4. Because both the correct and the erroneous paths start from the same state $\left(S_{0}=S_{0}^{\prime}\right), S_{t}$ and $S_{t}^{\prime}$ belong to the same level $t$ group.

5. Since $b_{0} \neq b_{0}^{\prime}, S_{t}$ and $S_{t}^{\prime}$ belong to different subgroups of the same level $t$ group.

The first 3 facts describe the relationship between the encoder states and the groups and subgroups at different levels. In the case of the ST code of Figure 1, the level 1 groups consist of 4 consecutive states, starting at state $0,4,8$ and 12 . The subgroups consist of only one state. The only level 2 group is comprised of all the 16 states, and its subgroups are made up of 4 consecutive states, starting at state $0,4,8$ and 12 .

Fact 4 states that if the $m$ th indices of the channel symbol index vectors at states belonging to any level $t$ group are the same, then the $m$ th entry of the $t$ th column of $\mathbf{B}_{\mathbf{1}}$ will be zero since, at state transition $t$, both the correct and the erroneous paths go through states that belong to the same level $t$ group. For example, if we take a look at the $S_{1} \rightarrow S_{2}$ state transition in Figure 1, state 8 and 11 belong to the same level 1 group, and the 2nd indices of the channel symbol index vectors $[0,0,1]^{T}$ and $[1,2,1]^{T}$ are the same. As a consequence, the 1 st column of the $\mathbf{B}_{1}$ matrix becomes $[1-j, 2,0]^{T}$.
The last fact can be interpreted as follows: if the $m$ th indices of the channel symbol index vectors at states belonging to different subgroups of the same level $t$ group are different, then the $m$ th entry of the $t$ th column of $B_{1}$ will be nonzero since, at state transition $t$, the correct and the erroneous paths go through states that belong to different subgroups of the same level $t$ group. To continue the example with the $S_{2} \rightarrow S_{3}$ state transition, state 0 and 13 belong to different subgroups of the same (only) level 2 group. The 2nd indices of the channel symbol index vectors $[0,0,0]^{\mathrm{T}}$ and $[0,1,3]^{T}$ are different, so the 2 nd column of the matrix $\mathbf{B}_{\mathbf{1}}$ will be $[0,1-j, 1+j]^{T}$.

Putting the previously described pieces together, we can state the second half of the design criteria:

(2a) For $t=1,2, \ldots, K-1$, the $t$ th indices of the channel symbol index vectors at states belonging to the same subgroup of any level $t$ group must be the same, and they must be different from the $t$ th indices of the channel symbol index vectors at states belonging to any other subgroup of that group.

(2b) For $t=1,2, \ldots, K-2$, the $(t+1) \mathrm{st},(t+2) \mathrm{nd}, \ldots$, $(K-1)$ st indices of the channel symbol index vectors at states belonging to the same level $t$ group must be the same. (Note that criterion (2b) is omitted for $t=K-1$.)

Having made the matrix $\mathbf{B}_{\mathbf{1}}$ full rank, the final task is to show that the channel symbol difference matrix $\mathbf{B}$ corresponding to the transmission of all $T$ source symbols is also of full rank. The matrix $\mathbf{B}$ can be decomposed as:

$$
\mathbf{B}=\left[\mathbf{B}_{\mathbf{1}}, \mathbf{B}_{\mathbf{2}}\right]
$$

where $\mathbf{B}_{\mathbf{1}}$ is as defined above and $\mathbf{B}_{\mathbf{2}}$ is a $K$ by $(T-K)$ matrix. Since $\mathbf{B}_{\mathbf{2}}$ is arbitrary, this description includes the cases when the correct and the decoded paths diverge and merge several times. From linear algebra, it is well known that if $\mathbf{B}_{\mathbf{1}}$ is of full rank, then $\mathbf{B}$ is also of full rank. Consequently, the design rules will produce codes that provide full diversity advantage.

\section{SIMULATION RESULTS}

To illustrate the performance of the codes designed using the above described method, we show some simulation results. The simulated communication system had one receive antenna. The source symbols were transmitted in frames of length 130 , and the Viterbi algorithm with decoding depth of 20 state transitions was used to decode the received signals. For each frame, the path gains between the transmit antennas and the receive antenna were modeled as independent, complex, zero mean, circularly symmetric Gaussian random variables with unit variance.

Since the frame error probability depends on the length of the frame and it does not seem very informative, we present probability of bit error curves as functions of the average signal to noise ratio (SNR) per source symbol at the receive antenna. In the sequel, the expression coding gain will refer to the difference (in $\mathrm{dB}$ ) of transmit energies to achieve the same probability of bit error value.

The repetition coded delay diversity of [3] is a special case of our design rules. Figure 2 shows the performance of this scheme for different number of transmit antennas ( $K=2,3,4$ and $N=$ $4,16,64$, respectively) and QPSK modulation $(b=2)$. It can be observed that the codes indeed provide different spatial diversity advantages since the steepness of the bit error rate curves is different. 


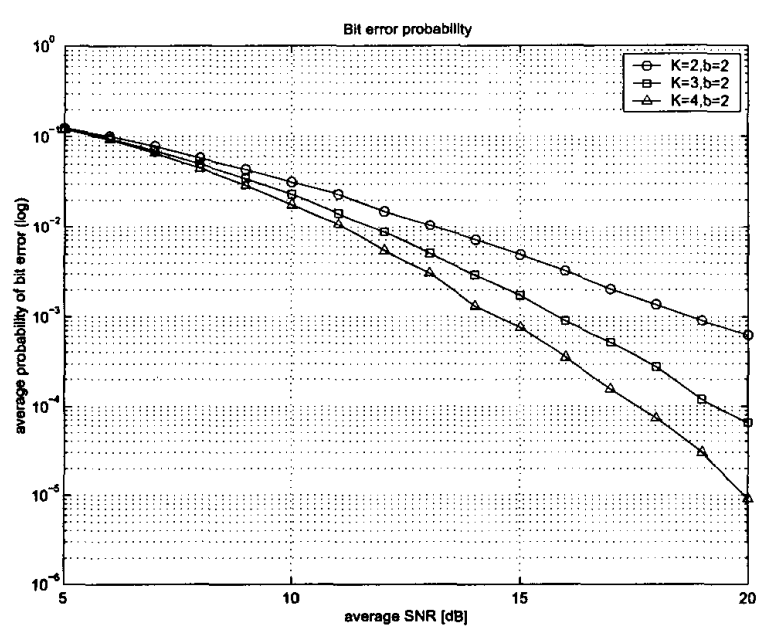

Fig. 2. Delay diversity ST codes, QPSK

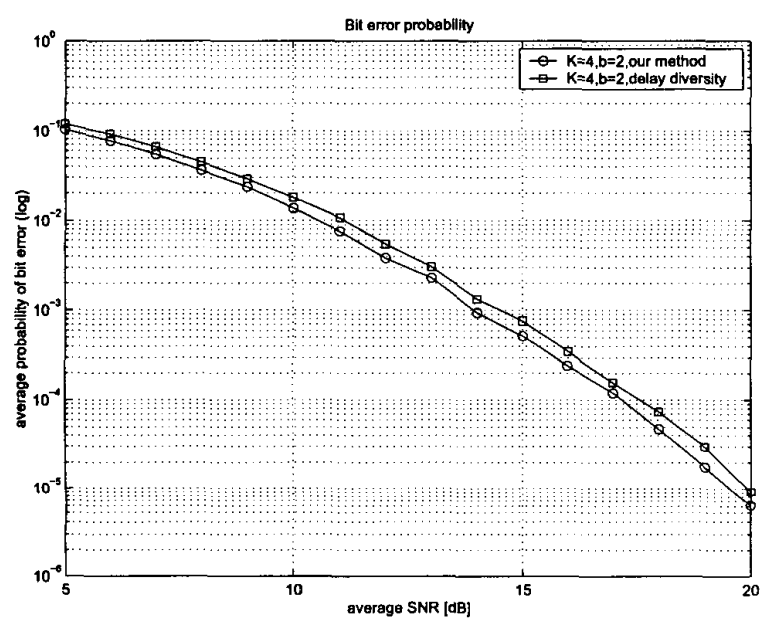

Fig. 3. ST codes for 4 antennas, QPSK

The two other figures compare the performance of the delay diversity construction and our approach. Our codes were optimized for coding advantage. Figure 3 depicts the results for 4 transmit antennas ( $K=4, N=64$ ) and QPSK modulation $(b=2)$. The two probability of bit error curves are shifted versions of each other, as expected. The minimum determinants of the delay diversity scheme and our method are $\gamma_{1}=16$ and $\gamma_{2}=32$, respectively. These numbers predict a relative coding advantage of $\sqrt[K]{\gamma_{2} / \gamma_{1}}=1.19$, so the observed approximately $0.5 \mathrm{~dB}$ coding gain over the delay diversity scheme is not surprising.

Figure 4 shows the bit error rate curves of a 3 transmit antenna system $(K=3, N=64)$ with 8PSK modulation $(b=3)$. At low SNR, the two error performance curves are close to each other, and they behave according to the expectations only at middle and high SNRs. This phenomenon may be due to the fact that at low SNR, the large number of transmission errors and the small minimum distance of the constellation prevent the Viterbi algorithm

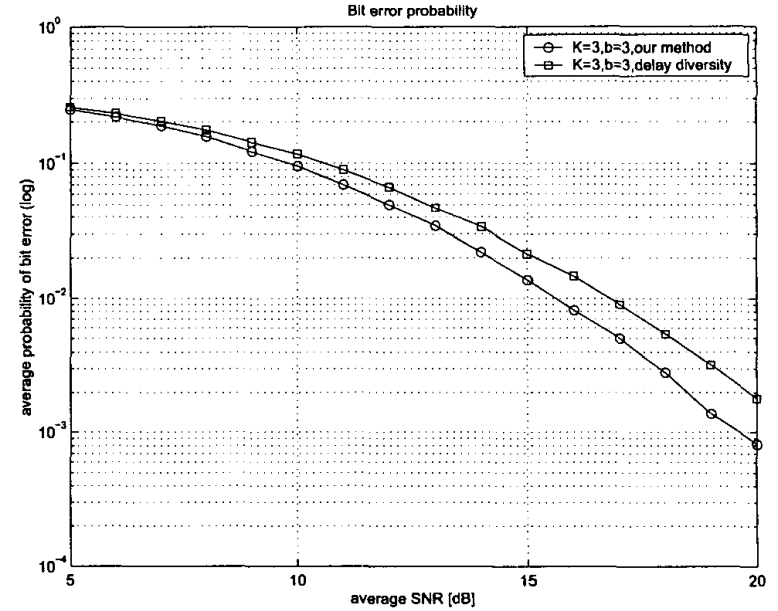

Fig. 4. ST codes for 3 antennas, 8PSK

with finite decoding depth from working properly. Moreover, the definition of coding advantage [2] is based on an upper bound on the $Q(x)$ Gaussian tail probability function, and this bound is not tight at low SNR. The minimum determinant of the delay diversity scheme is $\gamma_{1}=0.2010$, and the minimum determinant of our ST code is $\gamma_{2}=0.6863$, so the relative coding advantage becomes $\sqrt[K]{\gamma_{2} / \gamma_{1}}=1.51$. The simulation also shows that the performance improvement is more pronounced: at higher SNR, more than $1 \mathrm{~dB}$ coding gain can be achieved.

\section{CONCLUSION}

Having observed the group/subgroup structure of the state transitions, we proposed systematic design rules for ST trellis codes that achieve full spatial diversity. The simulation results demonstrate that the remaining freedom in the design space can be used to improve the performance. As the size of the constellation increases, the additional optimization for coding advantage becomes more important.

\section{REFERENCES}

[1] J. Guey, M. Fitz, M. Bell, and W. Kuo, "Signal design for transmitter diversity wireless communication systems over rayleigh fading channels," IEEE Vehicular Technology Conference, pp. 136-140, 1996.

[2] V. Tarokh, N. Seshadri, and A. Calderbank, "Space-time codes for high data rate wireless communication : Performance criterion and code construction," IEEE Transactions on Inf. Theory, vol. 44, no. 2, pp. 744-765, March 1998.

[3] J. Grimm, M. Fitz, and J. Krogmeier, "Further results on space-time coding for rayleigh fading," Proceedings of the 36 th Allerton Conference on Communications, Control and Computing, pp. 391-400, 1998.

[4] A. Hammons and H. El Gamal, "On the theory of space-time codes for psk modulation," IEEE Transactions on Inf. Theory, vol. 46, no. 2, pp. 524-542, March 2000. 